\title{
Monitoreo de movimientos de ladera en el sector de San Pedro de Vilcabamba mediante procedimientos GPS
}

\author{
Belizario A. Zárate Torres \\ Unidad de Ingeniería Civil, Geología y Minas, Escuela de Ingeniería Civil, Universidad Técnica \\ Particular de Loja, Loja, Ecuador \\ Autor para correspondencia: bazarate@utpl.edu.ec \\ Fecha de recepción: 25 de julio de 2011 - Fecha de aceptación: 20 de diciembre de 2011
}

\begin{abstract}
RESUMEN
Los movimientos de ladera a menudo afectan grandes zonas y muchas de las veces pasan desapercibidos por la lentitud de su dinámica, notándose únicamente evidencias geomorfológicas de su actividad, tales como grietas en el suelo que pueden ser el reflejo de otros procesos. Por las lluvias extremas los movimientos de ladera pasivos pueden convertirse rápidamente en activos causando pérdidas económicas y de vidas humanas. En Ecuador no existe un control de los movimientos de ladera que permita tener los datos necesarios para predecir su comportamiento con el tiempo. De acuerdo a publicaciones internacionales, la utilización de la técnica de monitoreo por GPS ha permitido conocer los límites, tamaño, nivel de actividad, dirección y velocidad de desplazamiento. En esta publicación, la técnica fue aplicada para cuantificar el movimiento de una ladera en el sector de San Pedro de Vilcabamba, en el sur del Ecuador.
\end{abstract}

Palabras clave: Movimientos de ladera, precipitación, geomorfología, GPS, velocidad de desplazamiento.

\begin{abstract}
Landslides often affect large areas, developing latent due to the slowness of the internal dynamics and in most cases only notable by geomorphologic evidences such as soil fissures, which in several cases can be the reflection of other processes. Triggered by extreme rainfall passive potential landslides can convert quickly to active landslides causing huge economic losses, which can be accompanied by loss of human life. Landslide control, consisting in the collection of data to predict the behavior of slopes in time, does not yet exist in Ecuador. According to international literature, surveying the activity of the surface of potential landslide areas using GPS as monitoring technique permits to understand the limits, size, level of activity, direction and velocity of earth movement. In this paper, the technique was applied to quantify the movement of a landslide area in the San Pedro sector of Vilcabamba, southern Ecuador.
\end{abstract}

Keywords: Landslide, precipitation, geomorphology, GPS, displacement velocity.

\section{INTRODUCCIÓN}

Uno de los principales problemas que se ha observado en la ciudad de Loja y porque no decirlo a nivel del Ecuador en relación a movimientos de ladera, es la falta de un monitoreo constante que permita obtener datos que describan el movimiento del terreno y con ello determinar las velocidades de desplazamiento como también el comportamiento a largo plazo. Conociendo la velocidad de un deslizamiento, es posible categorizar su actividad en activo o inactivo. Aparentemente un movimiento de ladera puede estar inactivo hasta que no se presenten otros factores que influyan en el comportamiento del mismo, como son: sismo, lluvia, cambio en el nivel freático, etc. El conocimiento 
de los desplazamientos que ocurren en un movimiento de ladera permite establecer un sistema de control ante posibles desastres que puedan ocurrir y establecer las medidas de mitigación a tiempo.

La determinación cinemática de una masa de terreno está basada en procedimientos geotécnicos y geodésicos (Acar y col., 2008; Cornejo, 2004), en estos últimos, los comúnmente usados para el monitoreo de deslizamientos son los distanciómetros láser y las estaciones totales; con ellos, los intervalos de adquisición de datos puede ser en forma diaria, semanal y mensual. Dentro de las técnicas empleadas para el registro de datos de un deslizamiento en campo geotécnico se puede mencionar el uso de extensiómetros y deformímetros, que son usados para determinar los desplazamientos superficiales. Estas técnicas se han empleado con mucho éxito en países como Colombia, Eslovaquia y Perú. Con el avance de la tecnología, aparecen nuevos dispositivos que se emplean para el monitoreo de laderas, entre los cuales se puede mencionar la Telemetría Láser por Scanner (SLR) y el Sistema de Posicionamiento Global (GPS).

La técnica de monitoreo con GPS ha permitido registrar movimientos de ladera logrando obtener buenos resultados; es una técnica práctica en la que únicamente es necesario conocer las coordenadas de un punto en función del tiempo. Éste sistema de monitoreo permite obtener precisiones de hasta milímetros al igual que se lo puede obtener con métodos topográficos convencionales (Tomas y col., 2005); la técnica antes mencionada, ha permitido medir los deslizamientos con la suficiente precisión que los métodos tradicionales topográficos (Zhou y col., 2005). Si se estableciese un sistema de coordenados tridimensional, las variaciones positivas como negativas de las diferencias de posición permiten obtener la componente de dirección: latitud, longitud y altitud del punto analizado y determinar el vector resultante de dirección; sin embargo, un monitoreo geodésico satelital debe ser complementado por la investigación geotécnica con el fin de obtener datos confiables en la determinación de las causas que inducen a un movimiento de ladera.

En el sector de San Pedro de Vilcabamba existe un deslizamiento que afecta un área aproximada de 4 hectáreas y que su movimiento es imperceptible a simple vista; sin embargo, vestigios de actividad se pueden observar en la base del deslizamiento lo que ha provocado daños en la carretera y viviendas cercanas. Ante ello se pretendió, con el uso de la técnica de GPS diferencial obtener las coordenadas de cada uno de los puntos de monitoreo instalados en el área de estudio y luego del post procesamiento de la información establecer la variación de las coordenadas lo que determinó las componentes del movimiento y su velocidad en el tiempo que duró el monitoreo.

\section{CLIMA Y GEOLOGÍA}

La parroquia rural de San Pedro de Vilcabamba se encuentra ubicada a $33 \mathrm{~km}$ de la ciudad de Loja. El acceso a esta población se la realiza mediante una vía de segundo orden que une las poblaciones de Loja, Malacatos, San Pedro de Vilcabamba, Vilcabamba, Yangana, Valladolid y Zumba; estas dos últimas, correspondientes a la Provincia de Zamora Chinchipe. El clima es subtropical templado con una temperatura que fluctúa entre los $18,7^{\circ} \mathrm{C}$ (mínima en el mes de julio) y $19,9^{\circ} \mathrm{C}$ (máxima en el mes de noviembre), con un promedio de $19,4^{\circ} \mathrm{C}$. En la zona de estudio está bien definida la estación lluviosa y la de verano; la primera se inicia entre octubre o noviembre hasta mayo, mientras que el verano comienza desde junio a septiembre. La precipitación anual en el valle es de $800 \mathrm{~mm}$.

Geológicamente, esta zona se encuentra al Este de la cuenca sedimentaria de Vilcabamba correspondiente a la formación Quillollaco de la edad del Mioplioceno de la era Terciaria, compuesta básicamente de conglomerados de arena gruesa, areniscas y limonitas; el conglomerado tiene un color café-amarillo con componentes bien redondeados de 2 a $30 \mathrm{~cm}$ de diámetro. Descansa sobre la formación San Cayetano correspondiente a la edad del Mioceno y se encuentra conformada por lutitas, limonitas, arcillas y conglomerados; también se puede encontrar en esta formación, manto de carbón y tobas. Se estima que el espesor de la formación es de $700 \mathrm{~m}$.

La formación de movimientos de ladera genera una serie de cambios en las características de los suelos y de la forma del relieve. Estos cambios que son visibles en el terreno son los denominados "Indicadores antecedentes". Los principales mecanismos de la inestabilidad detectados alrededor de la 
zona de estudio son: flujos de barro y deslizamientos; estos están compuestos por suelo orgánico con un alto contenido de agua. La Cinemática de estos flujos es muy variable y depende de algunos factores, entre ellos se puede mencionar: propiedades geotécnicas del suelo, contenido de agua, entre otras. En la mayoría de estos flujos se pudo establecer que los mismos se produjeron en las zonas de desembocadura de microcuencas y por la velocidad que presentan arrastran consigo partículas de material más grandes dentro de la masa de flujo.

Mediante la observación directa de la zona del movimiento de ladera, se puede evidenciar la presencia de algunos indicadores potenciales que demuestran la actividad del deslizamiento (Alcántara, 1999), aquellos indicadores se catalogan en grietas, escarpes y lobulaciones. En la superficie del deslizamiento es muy notoria la formación de escarpes cuyo desnivel con respecto a la zona estable es del orden de $60 \mathrm{~cm}$. Igualmente, se puede observar grietas en toda el área, muchas de ellas se encuentran cubiertas con vegetación, su ancho varía desde 5 hasta $20 \mathrm{~cm}$ y su profundidad es variada.

La presencia de lóbulos, pequeños escarpes y agrietamientos en el suelo, son visibles en zonas muy próximas al pie del deslizamiento. La existencia de depresiones (Mendoza y col., 1996) en esta zona hace que la escorrentía superficial, producto de las lluvias, se acumule y produzca la saturación del suelo; ante ello, algunas zonas pantanosas y lagunas están siendo drenadas mediante canales construidos a mano y que desembocan a quebradas naturales existentes en el sitio; con el fin de evitar la saturación del suelo, la expansión de la laguna y el deterioro del suelo cultivable. En la Figura 1 se puede observar los lugares donde existen flujos y deslizamientos como también lugares donde se presentan las evidencias geomorfológicas antes descritas.

Los deslizamientos son los principales mecanismos de inestabilidad que existen en la zona y uno de ellos, cuya área de afectación es de aproximadamente 4 hectáreas, se constituye en el de mayor extensión. Existen otros que en el transcurso de los años se han producido principalmente por la saturación del suelo ocasionado por fuertes tormentas en la zona y por infiltración de agua proveniente de canales de riego.

\section{METODOLOGÍA}

El monitoreo de deslizamientos se basa en el principio de determinar los cambios de distancias, alturas, ángulos o las coordenadas relativas de puntos de control (monumentos) distribuidos en la zona de estudio (Abidin y col., 2004). Existen varias técnicas que permiten obtener información relacionada al movimiento de una ladera y se resumen en la Tabla 1 adoptada y mejorada por Gili y col., (2000). Para el monitoreo del movimiento de ladera de San Pedro de Vilcabamba se utilizó la técnica de monitoreo con GPS. El Sistema de Posicionamiento Global, es un método tipo pasivo, basado en la navegación y posición de los satélites, puede ser operado durante el día y la noche, en tiempo soleado o durante la lluvia y no requiere de líneas visuales entre estaciones (Wolf y col., 2008). Es una técnica que no solo es utilizada para fines científicos sino que también puede ser utilizada para determinar deformaciones y fallas en estructuras (Huang y col., 2007; Xialo y col., 2002).

Para la obtención de datos precisos es necesario utilizar un procedimiento denominado DGPS (Differential GPS), que incluye el uso simultáneo de dos o más receptores basados en código. En este régimen, un receptor ocupa un punto denominado estación, base del cual se conocen las coordenadas precisas y el otro receptor o receptores denominados también "rovers" ocupan las estaciones de interés y cuyas coordenadas se desconocen. El principio para establecer movimientos de ladera utilizando el procedimiento DGPS se basa en obtener una variación matemática de las coordenadas de un punto (estación) dentro de un rango de tiempo. La posición relativa se la obtiene mediante la diferencia entre la última y la primera medición; este rango es variable y va desde días, meses a años. 


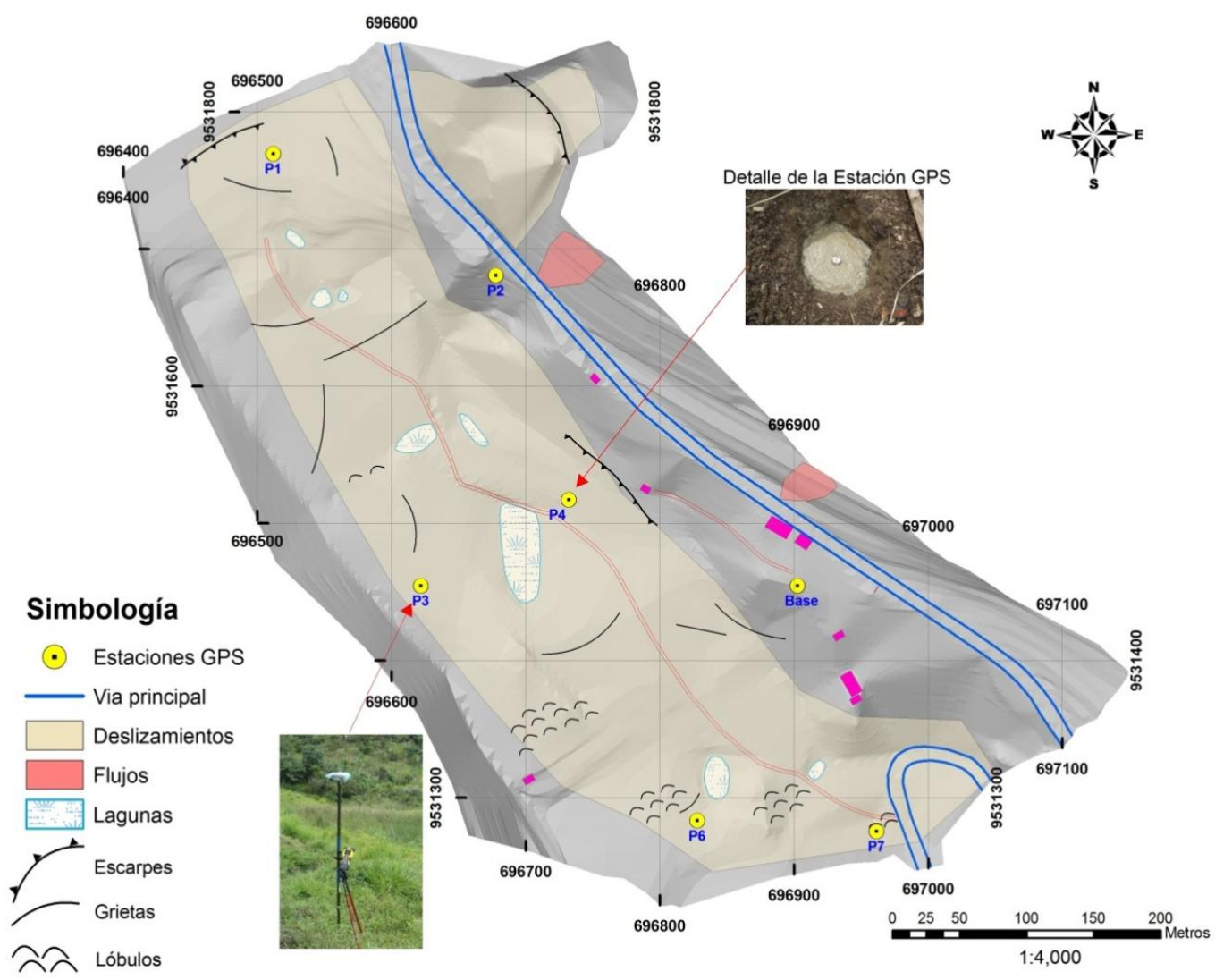

Figura 1. Mapa de distribución de estaciones GPS, Inventario de deslizamientos y geomorfología.

Tabla 1. Métodos y técnicas de medición para deslizamientos (en base a Gili y col., 2000).

\begin{tabular}{|c|c|c|c|}
\hline Método/Técnica & Resultados & Rango Típico & Precisión Típica \\
\hline Cinta de precisión & Cambio de distancia & $<30 \mathrm{~m}$ & $0,5 \mathrm{~mm} / 30 \mathrm{~m}$ \\
\hline $\begin{array}{l}\text { Extensómetro de cable } \\
\text { fijo }\end{array}$ & Cambio de distancia & $<10-80 \mathrm{~m}$ & $0,3 \mathrm{~mm} / 30 \mathrm{~m}$ \\
\hline $\begin{array}{c}\text { Varilla para la apertura } \\
\text { de grietas }\end{array}$ & Cambio de distancia & $<5 \mathrm{~m}$ & $0,5 \mathrm{~mm}$ \\
\hline $\begin{array}{l}\text { Desplazamiento desde el } \\
\text { inicio }\end{array}$ & $\begin{array}{l}\text { Diferencia de coordenadas } \\
\text { (2D) }\end{array}$ & $<100 \mathrm{~m}$ & $0,5-3 \mathrm{~mm}$ \\
\hline Triangulación & $\begin{array}{c}\text { Diferencia de coordenadas } \\
\text { (2D) }\end{array}$ & $<300-1000 \mathrm{~m}$ & $5-10 \mathrm{~mm}$ \\
\hline Oblicuo/polígono & $\begin{array}{l}\text { Diferencia de coordenadas } \\
\text { (2D) }\end{array}$ & $\begin{array}{l}\text { Variable, usualmente } \\
<100 \mathrm{~m}\end{array}$ & $5-10 \mathrm{~mm}$ \\
\hline Nivelación & Cambio de altura & $\begin{array}{l}\text { Variable, usualmente } \\
<100 \mathrm{~m}\end{array}$ & $2-5 \mathrm{~mm} \mathrm{~km}^{-1}$ \\
\hline Precisión de nivelación & Cambio de altura & $\begin{array}{l}\text { Variable, usualmente } \\
<50 \mathrm{~m}\end{array}$ & $0,2-1,0 \mathrm{~mm} \mathrm{~km}^{-1}$ \\
\hline $\begin{array}{c}\text { EDM (Medición } \\
\text { electrónica de distancia) }\end{array}$ & Cambio de distancia & $\begin{array}{c}\text { Variable, usualmente } \\
1-14 \mathrm{~km}\end{array}$ & $1-5 \mathrm{~mm}+1-5 \mathrm{ppm}$ \\
\hline Fotogrametría terrestre & $\begin{array}{l}\text { Diferencia de coordenadas } \\
\text { (3D) }\end{array}$ & $\begin{array}{l}\text { Idealmente } \\
<100 \mathrm{~m}\end{array}$ & $\begin{array}{l}20 \mathrm{~mm} \text { desde } \\
100 \mathrm{~m}\end{array}$ \\
\hline Fotogrametría aérea & $\begin{array}{l}\text { Diferencia de coordenadas } \\
\text { (3D) }\end{array}$ & $\mathrm{H}$ volado $<500 \mathrm{~m}$ & $10 \mathrm{~cm}$ \\
\hline $\begin{array}{l}\text { Clinómetro } \\
\text { Teodolito de precisión }\end{array}$ & $\begin{array}{l}\text { Cambio de ángulo } \\
\text { Cambio de ángulo }\end{array}$ & $\begin{array}{l} \pm 10^{\circ} \\
\text { Variable }\end{array}$ & $\begin{array}{l} \pm 0,01-0,1^{\circ} \\
\pm 10^{\circ}\end{array}$ \\
\hline Monitoreo con GPS & $\begin{array}{l}\text { Diferencia de coordenadas } \\
\text { (3D) }\end{array}$ & Variable & $2-5 \mathrm{~mm}+1-2 \mathrm{ppm}$ \\
\hline
\end{tabular}




\subsection{Aplicación de la técnica}

El periodo de monitoreo por el procedimiento de DGPS, se inició el 07 de julio de 2010 y se concluyó el 15 enero de 2011, la configuración de la red de monitoreo es la indicada en la Figura 1. Tanto la estación base como las estaciones de monitoreo se construyeron in situ y constaron de un mojón de hormigón de 1,20 $\mathrm{m}$ de profundidad y en cuyo centro se instaló una varilla de acero corrugado con un diámetro de $14 \mathrm{~mm}$; en el extremo libre de la varilla se realizó una pequeña perforación en su centro de $3 \mathrm{~mm}$ de diámetro donde se acopló la punta del bastón del receptor GPS. Las estaciones de monitoreo GPS fueron ubicadas estratégicamente en zonas donde hay la presencia de escarpes, lóbulos y grietas, a fin de establecer el desplazamiento de la estación. Por tratarse de un procedimiento DGPS, la estación base se implantó en una zona donde no se advierte cambios geomorfológicos; además, estuvo localizada en una zona elevada para tener una mejor cobertura espacial. La distancia entre la base y las estaciones de monitoreo osciló entre 180 y 505 m. Se utilizó receptores GPS de doble frecuencia Trimble R6. Las mediciones fueron almacenadas con un intervalo de 15 segundos y se calibró el equipo para una máscara de elevación de $15^{\circ}$. Los datos GPS fueron colectados en modo Fast Static usando un receptor y un móvil, el tiempo de permanencia del móvil en cada estación de monitoreo fue de 30 min (Acar, 2010; Abidin y col., 2004; Liu y col., 2004; Setal y col., 2006; Tagliavini y col., 2007).

\subsection{Procesamiento de datos}

El procesamiento de los datos de GPS tanto de la base como de los puntos de monitoreo fueron analizados mediante el Software Trimble Business Center Versión 2.2. Dentro de los parámetros considerados para el post procesamiento se encuentran: Sistema de coordenadas: UTM, zona: 17 Sur; transformación de datum: WGS 1984. Como criterios adicionales considerados para aceptar el post procesamiento de los datos se tomó como indicador aceptable: $0,005 \mathrm{~m} \pm 0,5 \mathrm{ppm}$ (ppm: partes por millón) y para no aceptar la precisión: $0,015 \mathrm{~m} \pm 0,5 \mathrm{ppm}$. Luego de efectuado el post procesamiento de los datos se obtuvieron las coordenadas precisas de cada uno de los puntos de monitoreo y con ello se calculó la variación entre coordenadas, como se lo muestra en la Tabla 2.

Tabla 2. Coordenadas de los puntos de monitoreo y desplazamientos.

\begin{tabular}{|c|c|c|c|c|c|c|c|c|c|}
\hline \multirow{3}{*}{$\begin{array}{l}\text { Periodo } \\
\text { Estación }\end{array}$} & \multicolumn{3}{|c|}{ Julio 2010} & \multicolumn{3}{|c|}{ Enero 2011} & \multirow{2}{*}{\multicolumn{3}{|c|}{$\delta$ (Desplazamiento) }} \\
\hline & \multicolumn{3}{|c|}{ Coordenadas iniciales } & \multicolumn{3}{|c|}{ Coordenadas finales } & & & \\
\hline & $\mathrm{N}(\mathrm{m})$ & $\mathrm{E}(\mathrm{m})$ & $\mathrm{Z}(\mathrm{m})$ & $\mathrm{N}(\mathrm{m})$ & $\mathrm{E}(\mathrm{m})$ & $\mathrm{Z}(\mathrm{m})$ & $\delta_{N}(\mathrm{~m})$ & $\delta_{E}(\mathrm{~m})$ & $\delta_{Z}(\mathrm{~m})$ \\
\hline Base & 9531453,633 & 696902,627 & 1689,986 & 31453,633 & 696902,627 & 1689,986 & 0 & 0 & 0 \\
\hline P1 & 9531769,727 & 696511,789 & 1714,941 & 9531768,650 & 696512,799 & 1714,551 & $-1,077$ & 1,010 & $-0,390$ \\
\hline $\mathrm{P} 2$ & 9531680,892 & 696677,727 & 1716,723 & 9531680,893 & 696677,727 & 1716,723 & 0,001 & 0,000 & 0,000 \\
\hline $\mathrm{P} 3$ & 9531454,749 & 696621,887 & 1686,013 & 9531453,747 & 696621,707 & 1686,039 & $-1,002$ & $-0,180$ & 0,026 \\
\hline $\mathrm{P} 4$ & 9531517,421 & 696732,033 & 1679,536 & 9531516,207 & 696731,858 & 1679,193 & $-1,214$ & $-0,175$ & $-0,343$ \\
\hline P6 & 9531283,344 & 696827,931 & 1658,322 & 9531283,350 & 696827,920 & 1658,309 & 0,006 & 0,000 & $-0,013$ \\
\hline P7 & 9531275,640 & 696961,484 & 1643,351 & 9531275,641 & 696961,518 & 1643,386 & 0,001 & 0,034 & 0,035 \\
\hline
\end{tabular}

Para analizar los desplazamientos horizontales $\delta_{E-N}$, se calculó un vector resultante con las componentes $\delta_{E}$ y $\delta_{N}$ (Sdao y col., 2005) de cada punto monitoreado con la siguiente expresión:

$$
\delta_{E-N}=\sqrt{\delta_{E}^{2}+\delta_{N}^{2}}
$$

donde $\delta_{E}$ y $\delta_{N}$ es la diferencia de coordenadas Este y Norte respectivamente, correspondiente a los meses de julio 2010 y enero 2011. 
La prueba de congruencia estadística se llevó a cabo con cada vector $\delta_{E-N}$ calculado con la ecuación (1), lo que permitió comprobar estadísticamente los desplazamientos de cada punto (Abidin y col., 2004). Esta prueba también se aplicó a $\delta Z$ que es simplemente la diferencia de elevaciones de los puntos de monitoreo entre la primera y segunda campaña.

Esta comprobación se la realizó mediante el método de estimación por intervalos que consiste en la obtención de un intervalo dentro del cual estará el valor del parámetro estimado con una cierta probabilidad. Para la aplicación de este método se recurre a los siguientes parámetros:

$$
\begin{array}{lc}
\text { Límite de confianza }(1-\alpha): & 99 \% \\
\text { Nivel de significación }(\alpha): & 0,01 \\
\text { Valor crítico }\left(\mathrm{Z}_{\alpha / 2}\right): & 2,576
\end{array}
$$

Con los parámetros antes indicados se puede calcular el vector de desplazamiento máximo horizontal y vertical con un límite de confianza del $99 \% \Delta_{\operatorname{maxE}-\mathrm{N}}$ y $\Delta_{\operatorname{maxZ}}$ con las ecuaciones:

$$
\begin{gathered}
\Delta \max _{E-N}=Z_{\alpha / 2} \sqrt{E_{E}^{2}+E_{N}^{2}} \\
\Delta \max _{Z}=Z_{\alpha / 2} \sqrt{E_{Z}^{2}+E_{Z}^{2}}
\end{gathered}
$$

donde $E_{E}, E_{N}$ es el error estándar para deformaciones E-N que para el presente estudio se lo ha considerado de $1.5 \mathrm{~mm}$ y $\mathrm{E}_{\mathrm{Z}}$ es el error estándar para la deformación vertical cuyo valor considerado es $2 \mathrm{~mm}$ (Özer y col., 2010; Hartinger y col., 1999). Aplicando las ecuaciones (2) y (3) se puede establecer que $\Delta_{\operatorname{maxE}-\mathrm{N}}$ y $\Delta_{\max Z}$ son 0,005 y $0,007 \mathrm{~m}$ respectivamente.

Para establecer si existió movimiento en los puntos monitoreados es necesario verificar las siguientes condiciones:

$$
\begin{aligned}
& \mathrm{Si}, \delta_{E-N}>\Delta_{\operatorname{maxE}-\mathrm{N}} \text { entonces existe movimiento } \\
& \mathrm{Si}, \delta_{Z}<\Delta_{\max Z} \text { entonces no existe movimiento }
\end{aligned}
$$

Los desplazamientos $(\boldsymbol{\delta})$ son determinados por la diferencia de los valores de las coordenadas finales y las iniciales; al efectuar esta operación se obtienen valores positivos como negativos lo que indica la dirección de la componente sea esta, Norte, Sur, Este u Oeste. El vector $\delta_{E-N}$ corresponde al vector resultante de las componentes Norte y Este, y es a partir de este parámetro que se verifica las

\begin{tabular}{|c|c|c|c|c|c|c|c|c|}
\hline \multicolumn{5}{|c|}{$\delta$ (Desplazamiento) } & \multicolumn{4}{|c|}{ Análisis } \\
\hline \multirow{2}{*}{ Estación } & \multicolumn{2}{|c|}{$\delta(\mathrm{m})$} & \multicolumn{2}{|c|}{ Vector $(\mathrm{m})$} & \multirow{2}{*}{$\begin{array}{c}\text { Deformación } \\
\text { horizontal }\end{array}$} & \multirow{2}{*}{$\begin{array}{l}\text { Deformación } \\
\text { vertical }\end{array}$} & \multicolumn{2}{|c|}{ Deformación efectiva (m) } \\
\hline & $\delta_{N}$ & $\delta_{E}$ & $\delta_{Z}$ & $\delta_{N-E}$ & & & Vector E-N & Vector Z \\
\hline Base & 0,000 & 0,000 & 0,000 & 0,000 & $\begin{array}{c}\text { No hay } \\
\text { deformación }\end{array}$ & $\begin{array}{c}\text { No hay } \\
\text { deformación }\end{array}$ & 0,000 & 0,000 \\
\hline $\mathrm{P} 1$ & $-1,077$ & 1,010 & $-0,390$ & 1,476 & Deformación & Deformación & 1,471 & $-0,383$ \\
\hline $\mathrm{P} 2$ & 0,001 & 0,000 & 0,000 & 0,001 & $\begin{array}{l}\text { No hay } \\
\text { deformación }\end{array}$ & $\begin{array}{l}\text { No hay } \\
\text { deformación }\end{array}$ & 0,000 & 0,000 \\
\hline P3 & $-1,002$ & $-0,180$ & 0,026 & 1,018 & Deformación & Deformación & 1,013 & 0,019 \\
\hline $\mathrm{P} 4$ & $-1,214$ & $-0,175$ & $-0,343$ & 1,227 & Deformación & Deformación & 1,222 & $-0,336$ \\
\hline P6 & 0,006 & 0,000 & $-0,013$ & 0,006 & Deformación & Deformación & 0,001 & $-0,006$ \\
\hline P7 & 0,001 & 0,034 & 0,035 & 0,034 & Deformación & Deformación & 0,029 & 0,028 \\
\hline
\end{tabular}
condiciones antes indicadas; la deformación efectiva es definida como el verdadero desplazamiento del punto de monitoreo. Para su cálculo se establece la diferencia entre el vector resultante y el vector de desplazamiento máximo con el $99 \%$ de confianza; el cálculo de vectores de desplazamiento máximo horizontal y vertical, como también la verificación de las condiciones, se puede apreciar en la Tabla 3.

Tabla 3. Desplazamientos efectivos de los puntos de monitoreo. 


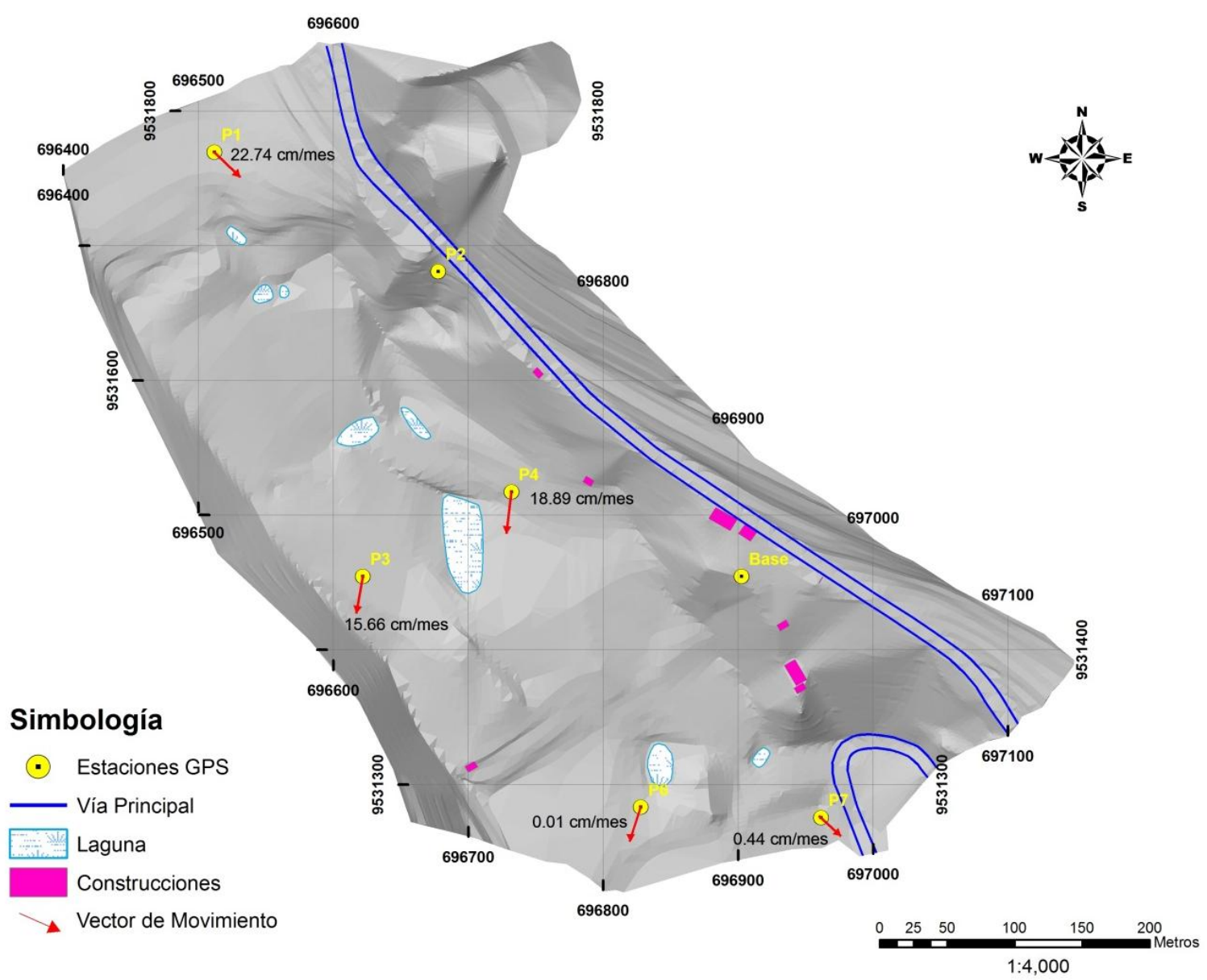

Figura 2. Mapa de vectores de velocidad y desplazamiento (velocidad en $\mathrm{cm} \mathrm{mes}^{-1}$ ).

\section{RESULTADOS}

La velocidad del movimiento de la ladera de San Pedro es determinado a partir de la deformación efectiva en cada punto de monitoreo y el tiempo transcurrido desde la primera campaña realizada el 07 de julio de 2010 hasta la última efectuada el 15 de enero de 2011; con ello, se estableció un tiempo transcurrido de 194 días (6,47 meses). Tomando como concepto que la velocidad es igual al desplazamiento dividido para el tiempo, se determinó para cada punto de control la velocidad del movimiento de ladera y con estos datos se elaboró un mapa de vectores de desplazamiento y de velocidad como se lo representa en la Figura 2. Tanto el punto P2 y la Base no registraron variación de coordenadas, por ende se encuentran en una zona estable con velocidad de desplazamiento cero. Los puntos P6 y P7 presentan una velocidad muy baja $\left(0,01\right.$ y $0,44 \mathrm{~cm} \mathrm{mes}^{-1}$ respectivamente) en relación al resto de puntos de control (P1, P3 y P4). Se puede observar en la Tabla 3, los puntos de control P1 y P4 son los que presentan mayor deformación horizontal y vertical, lo que indica que existe un cambio de posición de los puntos con relación a su estado inicial. Es notorio que la componente direccional $\delta_{N}$ del movimiento de ladera tiene una tendencia hacia el sur con una leve variación de la componente $\delta_{E}$ hacia el Oeste. En el punto P7 es evidente que existe un incremento en la cota respecto a la elevación tomada como referencia; en la Figura 1 este punto se encuentra en una zona con presencia de lobulaciones con lo que se comprueba la formación de éstas. 


\section{CONCLUSIONES}

El monitoreo con GPS aplicado al estudio de movimiento de laderas demuestra que ofrece la suficiente precisión milimétrica para medir la velocidad de desplazamiento de un movimiento de ladera aplicando el procedimiento DGPS (Differential Global Position System). El tiempo mínimo de permanencia del equipo en modo Fast Static de 30 min en cada punto a monitorear brinda la precisión suficiente para este tipo de trabajos; además, es una técnica que no requirió que exista una visual entre la base y las estaciones de monitoreo. Las estaciones de monitoreo P1, P3 y P4 son las que registran valores altos de velocidad y se puede apreciar que experimentaron también un descenso en su cota, lo que indica que toda esa masa de terreno está desplazándose hacia la base de deslizamiento. Tomando como referencia los puntos monitorizados que tienen mayor velocidad (rango de 15 a $23 \mathrm{~cm} \mathrm{mes}^{-1}$ ) permiten catalogar al movimiento como lento dentro en la Escala de Velocidades de ladera (Alcántara, 1999).

\section{AGRADECIMIENTOS}

El autor quiere dejar constancia de su agradecimiento a la Unidad de Ingeniería Civil Geología y Minas de la Universidad Técnica Particular de Loja, a la Universidad de Granada, España y en particular al Dr. Jan Feyen, por su apoyo y colaboración en el presente trabajo investigativo.

\section{BIBLIOGRAFÍA}

Acar, M., T. Ozludemir, S. Erol, N. Celik, T. Ayan, 2008. Kinematic landslide monitoring with Kalman filtering. Nat. Hazards Earth Syst. Sci., 8, 213-221.

Acar, M., 2010. Determination of strain accumulation in landslide areas with GPS measurements. Sci. Res. Essays, 5(8), 763-768.

Alcántara, I., 2000. Landslides: ¿Deslizamientos o movimientos del terreno? Definición, clasificaciones y terminología, investigaciones geográficas. Boletín del Instituto de Geografía, Universidad Nacional Autónoma de México, México, 41.

Ding, X., D. Huang, J. Yin, Y. Chen, C. Lau, Y. Yang, Y. Sun, W. Chen, X. He, 2003. Development and field testing of a multi-antenna GPS system for deformation monitoring. Wuhan University $J$. Nat. Sci., 8(2), 671-676.

Hartinger, H., F. Brunner, 1999. Development of a monitoring system of landslide motion using GPS. 9th FIG Symp. on Deformation Monitoring, Olsztyn, Poland, 29-38.

Huang, L., Y. Fu, 2007. Analysis on the noises from continuously monitoring GPS sites. Acta Seismologica Sinica, 20(2), 206-211.

Liu, G., Y. Zhu, R. Zhou, 2005. A new approach of single epoch GPS positioning for landslide monitoring. Acta Seismologica Sinica, id:1000-9116(2005)04-0427-08.

Özer, E., E. Mentese, 2007. Deformation network analysis. Istanbul Technical University, Istanbul, Turkey.

Pinggen Zhou, B., D. Jingjun Guo, F. Yanming, 2005. A demostrative GPS-aided automatic landslide monitoring system in Sichuan province. J. Global Posit. Syst., 4(1-2), 184-191.

Setal, H., R. Othman, 2006. Monitoring of offshore platform subsidence using permanent GPS stations. J. Global Posit. Syst., 5(1-2), 17-21.

Sdao, F., S. Pascale, P. Rutigliano, 2005. Geomorphological features and monitoring of a large and complex landslide near Avigliano urban area (South Italy). Adv. Geosci., 2, 97-101. 
Tagliavini, F., M. Mantovani, G. Marcato, A. Pasuto, S. Silvano, 2007. Validation of landslide hazard assessment by means of GPS monitoring technique: A case study in the Dolomites. Nat. Hazards Earth Syst. Sci., 7, 185-193.

Tomas, R., J. Delgado, J.M. Lopez-Sanchez, 2005. Técnicas de ingeniería cartográfica empleada en el estudio de subsidencia y movimientos de ladera: Principales características y análisis

comparativo. Congreso Internacional de Ingeniería Gráfica (INGEGRAF), Sevilla, España. 